\title{
Genetic Contributions to Altered Callosal Morphology in Schizophrenia
}

\author{
Katherine L. Narr, ${ }^{1,3}$ Tyrone D. Cannon, ${ }^{4}$ Roger P. Woods, ${ }^{2,3}$ Paul M. Thompson,,${ }^{1,3}$ Sharon Kim, ${ }^{1,3}$ \\ Dina Asunction, ${ }^{1,3}$ Theo G. M. van Erp, ${ }^{4}$ Veli-Pekka Poutanen, ${ }^{6}$ Matti Huttunen, ${ }^{7}$ Jouko Lönnqvist, ${ }^{7}$ \\ Carl-Gustav Standerksjöld-Nordenstam, ${ }^{6}$ Jaakko Kaprio, ${ }^{7,8}$ John C. Mazziotta, ${ }^{2,3,5}$ and Arthur W. Toga ${ }^{1,2,3}$ \\ ${ }^{1}$ Laboratory of Neuro Imaging, ${ }^{2}$ Ahmanson-Lovelace Brain Mapping Center, Neuropsychiatric Institute, ${ }^{3}$ Department of \\ Neurology, ${ }^{4}$ Departments of Psychology, Psychiatry, and Human Genetics, and ${ }^{5}$ Departments of Radiology and \\ Pharmacology, University of California at Los Angeles School of Medicine, Los Angeles, California 90095-1769, \\ ${ }^{6}$ Department of Radiology, Helsinki University Central Hospital, Meilahti Clinics, FIN-00290 Helsinki, Finland, ${ }^{7}$ Department \\ of Mental Health and Alcohol Research, National Public Health Institute of Finland, SF-0030 Helsinki, Finland, and \\ ${ }^{8}$ Department of Public Health, University of Helsinki, FIN-0014 Helsinki, Finland
}

Patients with schizophrenia exhibit abnormalities in midsagittal corpus callosum area, shape, and/or displacement. Our goal was to confirm these findings and to establish the genetic and nongenetic contributions to altered callosal morphology in schizophrenia. Relationships between ventricular enlargements potentially contributing to callosal displacements were assessed as a secondary goal. High-resolution magnetic resonance images were obtained from co-twins of monozygotic and dizygotic pairs discordant for schizophrenia and healthy control twins ( $N=40$ pairs). Investigators blind to group status segmented the corpus callosum and ventricles in native brain volumes aligned using a rigid-body transformation with no scaling. Total and parcellated midsagittal callosal areas and measures indexing vertical displacements of the corpus callosum were used in statistical tests to identify schizophrenia and sex effects and to dissociate genetic and nongenetic influences on morphology. Anatomical mesh modeling methods provided group average and surface variability maps of the callosum.
Callosal areas did not differ between groups defined by sex or biological risk. Vertical displacements of the callosum, pronounced in male patients, were confirmed in schizophrenia and observed between dizygotic, but not monozygotic co-twins discordant for schizophrenia. Like their affected twins, however, unaffected monozygotic co-twins of the schizophrenia probands exhibited significant callosal displacements. Lateral and third ventricle enlargements were related to callosal displacements. Results clearly support that genetic rather than diseasespecific or shared environmental influences contribute to altered callosal morphology in schizophrenia. An upward bowing of the callosum may thus provide an easily identifiable neuroanatomic marker to screen individuals possessing a biological vulnerability for schizophrenia.

Key words: corpus callosum morphology; schizophrenia; genetic effects; environmental effects; monozygotic twins; dizygotic twins; morphology; imaging
The corpus callosum, the major interhemispheric commissure composed of $\sim 3$ million myelinated fibers topographically connecting primarily homologous hemispheric regions, has been widely studied in schizophrenia. Abnormalities in the crosscallosal transfer of information (Woodruff et al., 1997; Mohr et al., 2000) (K. L. Narr, M. F. Green, L. Capettillo-Cunliffe, A. W. Toga, and E. Zaidel, unpublished observations) and differences in midsagittal callosal size are reported, although discrepancies in the direction of positive results and negative findings are present (Woodruff et al., 1995; Thompson et al., 2001a; Shenton et al.,

Received Nov. 14, 2001; revised Jan. 25, 2002; accepted Feb. 5, 2002.

This work was supported by National Library of Medicine Grant LM/MH05639, National Center for Research Resources (NCRR) Grant RR05056, and National Institute of Neurological Disorders and Stroke (NINDS) Grant NS38253, Human Brain Project grant to the International Consortium for Brain Mapping, funded by National Institute of Mental Health (NIMH), National Institute on Drug Abuse, National Cancer Institute, and NINDS Grant P20 MH/DA52176, by a P41 Resource Grant RR13642 from the NCRR, a Distinguished Investigator Award from the National Alliance for Research on Schizophrenia and Depression (J.C.M.), and a research grant from the NIMH (T.D.C.) that was used for data collection.

Correspondence should be addressed to Dr. Arthur W. Toga, Laboratory of Neuro Imaging, Department of Neurology, Division of Brain Mapping, University of California at Los Angeles, School of Medicine, 710 Westwood Plaza, Los Angeles, CA 90095-1769. E-mail: toga@loni.ucla.edu.

Copyright (C) 2002 Society for Neuroscience $\quad 0270-6474 / 02 / 223720-10 \$ 15.00 / 0$
2001). Reports of callosal surface displacements and/or shape differences appear more consistently in schizophrenia (Casanova et al., 1990a,b; DeQuardo et al., 1996; Frumin et al., 1998; Gharaibeh et al., 2000; Narr et al., 2000a). These defects may represent trait abnormalities, given their reported presence in first episode patients (Frumin et al., 1998; Gharaibeh et al., 2000).

Approximately seven studies have assessed schizophreniarelated callosal shape differences. Specifically, an upward bowing of the callosum was found in affected monozygotic co-twins compared with their unaffected co-twins (Casanova et al., 1990b), and associations between increased callosal curvature and ventricular enlargements were observed (Casanova et al., 1990a). DeQuardo et al. (1996) similarly reported focal midline abnormalities and a more arched corpus callosum in schizophrenia. Arching of the callosum was also apparent by visual inspection in first episode patients, although overall midline shape differences were not significant (Gharaibeh et al., 2000). Frumin et al. (1998) reported shape differences in the posterior callosum (increased curvature) in schizophrenia patients compared with controls and bipolar patients. Furthermore, we previously demonstrated vertical callosal surface displacements in schizophrenia that were linked with lateral ventricular enlargements (Narr et al., 2000a). 
Table 1. Demographic information for twin groups

\begin{tabular}{|c|c|c|c|c|}
\hline \multirow[b]{2}{*}{ Subjects } & \multicolumn{2}{|c|}{ Discordant co-twins } & \multicolumn{2}{|c|}{ Control co-twins } \\
\hline & $\mathrm{MZ}(n=20)$ & $\mathrm{DZ}(n=20)$ & $\mathrm{MZ}(n=20)$ & $\mathrm{DZ}(n=20)$ \\
\hline Sex, male/female & $10 \mathrm{~m} / 10 \mathrm{f}$ & $10 \mathrm{~m} / 10 \mathrm{f}$ & $10 \mathrm{~m} / 10 \mathrm{f}$ & $10 \mathrm{~m} / 10 \mathrm{f}$ \\
\hline Handedness, $\mathrm{N}$ left & 1 left & 0 left & 0 left & 1 left \\
\hline Substance abuse, $\mathrm{N}$ & 1 & 1 & 0 & 1 \\
\hline Cluster A disorder, $\mathrm{N}$ & 2 & 0 & 0 & 2 \\
\hline Age, mean (SD) & $48.3(2.9)$ & $49.0(3.9)$ & $48.3(3.8)$ & $47.9(4.2)$ \\
\hline Parental social class, mean (SD) & $4.6(0.8)$ & $4.2(1.7)$ & $4.0(1.0)$ & $4.4(0.8)$ \\
\hline Years cohabitation, mean (SD) & $20.7(3.4)$ & $19.9(3.6)$ & 22.7 (5.7) & 19.8 (2.9) \\
\hline
\end{tabular}

In contrast to the above findings, one study reported the callosum as bowed downwards in schizophrenia and schizotypal personality disorder (Downhill et al., 2000). Callosal shape, however, was measured after removing the callosa from the context of the midsagittal section. Significant relationships were still present between callosal displacement positions and ventricular enlargement, although displacements were not in the expected direction. Only one published study has failed to detect callosal shape differences in schizophrenia (Tibbo et al., 1998), in which landmarks chosen to identify callosal boundaries may have influenced results (DeQuardo, 1999).

The first goal of this study was to confirm earlier findings of callosal displacement in chronic schizophrenia, an effect that was more pronounced between male patients and controls than between female diagnostic groups (Narr et al., 2000a). Our main objective, however, was to characterize genetic and/or shared or disease-specific environmental contributions to altered midsagittal callosal morphometry in schizophrenia. Moreover, we wished to confirm whether lateral or third ventricular enlargements were associated with callosal displacements. To help clarify discrepancies among earlier results, we further aimed to establish whether callosal areas showed schizophrenia effects and genetic and/or nongenetic contributions, while noting that two earlier family studies have failed to support these influences. Specifically, no significant differences in callosal areas were found between monozygotic (MZ) co-twins discordant for schizophrenia (Casanova et al., 1990b), suggesting no disease-specific influences. Likewise, differences were not found between controls and first-degree relatives who were presumed obligate carriers of schizophrenia genes, failing to support genetic influences (Chua et al., 2000).

Based on data supporting callosal shape differences in schizophrenia, we hypothesized that displacements would be present: (1) in schizophrenia patients compared with controls irrespective of zygosity; and (2) in affected MZ co-twins compared with their healthy siblings (supporting nongenetic influences). We also examined, for the first time, differences in callosal morphology between healthy co-twins of schizophrenia probands and normal twins (supporting genetic liability and/or shared environmental influences). Here we hoped to identify a relatively simple endophenotype in imaging data that may be useful for screening in genetic linkage studies.

\section{MATERIALS AND METHODS}

Subjects. Subjects included 10 sets of monozygotic (MZ) and dizygotic (DZ) control twin pairs and $10 \mathrm{MZ}$ and $10 \mathrm{DZ}$ twin pairs discordant for schizophrenia $(N=80$ individuals). These subjects were randomly selected from a cohort of same-sex twins born in Finland from 1940 to 1957 (Cannon et al., 1998, 2002) with groups matched for age, sex, and other demographic criteria (Table 1). To determine the diagnostic status of each co-twin, two examiners blind to zygosity and diagnosis performed structured diagnostic interviews (reliability $=0.96 \pm 0.02$ ). Probands diagnosed with schizoaffective disorder and/or unaffected co-twins diagnosed with a psychotic disorder were excluded from the study. Exclusion criteria for control twin pairs included any personal and/or firstdegree relative family history of psychosis. MZ probands were equivalent to $\mathrm{DZ}$ probands in terms of age at evaluation, age at onset, positive symptom severity, and negative symptom severity (Cannon et al., 1998). DNA analysis with markers including DIS80 (20 alleles), DI7S30 (13 alleles), apoB (20 alleles), COL2A1 (10 alleles), vWA ( 9 alleles), and HUMTH01 (6 alleles), confirmed the zygosity of co-twins.

Image acquisition and analysis procedures. Three-dimensional (3-D) high-resolution T1-weighted Magnetization Prepared Rapid Acquisition Gradient Recalled Echo magnetic resonance (MR) images were obtained on the same Siemens 1.0 Tesla scanner (Siemens, New York, NY) in the Department of Radiology, Helsinki University Central Hospital. Series of 128 contiguous $1.2 \mathrm{~mm}$ sagittal brain slices were acquired $(256 \times 256$ matrix; repetition time, 11 ; echo time, $4.4 \mathrm{msec}$; field of view, 250; flip angle, $12^{\circ}$ ).

Figure 1 presents a flow chart summarizing all image processing steps. Each brain volume was corrected for magnetic field inhomogeneities (Zijdenbos and Dawant, 1994; Sled and Pike, 1998) and resliced into a standard orientation as follows. Ten standard anatomical landmarks were identified in all three planes and matched with a set of corresponding point locations defined on the ICBM-305 average brain (Mazziotta et al., 1995). These landmarks, identified in each image set by a trained operator (S.K.) blind to group status were then used to compute a threetranslation and three-rotation rigid-body linear transformation for each brain volume with no scaling (Sowell et al., 1999) using the software package Register developed by the Brain Imaging Center of the Montreal Neurological Institute (MacDonald et al., 1994; MacDonald, 1996). That is, each brain volume was reoriented to correct for head alignment and placed into the same coordinate system using trilinear interpolation and a six parameter Procrustes fit with no scaling. The 10 landmark points included, bilaterally: (1-2) the apex of the triangle formed by the transverse sinus, cerebrum, and cerebellum in the coronal plane after the disappearance of the horizontal striations of the cerebellum in the sagittal plane; (3-4) the center of the eye sockets where eye socket bone diameters were largest in all three planes; (5) the most anterior points of the temporal lobe; (6) unilaterally the most anterior point of the genu of the corpus callosum, in the midsagittal plane defined by presence of the falx cerebri, septum pellucidum, and the interhemispheric fissure; (7) the most posterior point of the corpus callosum at the bulge of the splenium in the midsagittal plane; (8) the apex of the fourth ventricle in the midsagittal view; (9) the most posterior point of the fourth ventricle in the axial view; and (10) the center of the mammillary bodies in the midsagittal plane (Sowell et al., 1999). Image volumes were thus resampled into $1 \mathrm{~mm}$ isotropic voxels and placed into the standard coordinate system of the ICBM-305 average brain (Mazziotta et al., 1995), correcting only differences in brain alignment between image volumes. The centroid location of these 10 landmarks was mapped to the same coordinate location for each data set.

Corpus callosum delineation. The midsagittal sections from each brain volume were brought into register by the 10 point registration described above and verified by confirming the presence of the falx cerebri, septum pellucidum, and the vertical orientation of the interhemispheric fissure in all three planes. One rater (K.N.) blind to group status traced the corpus 


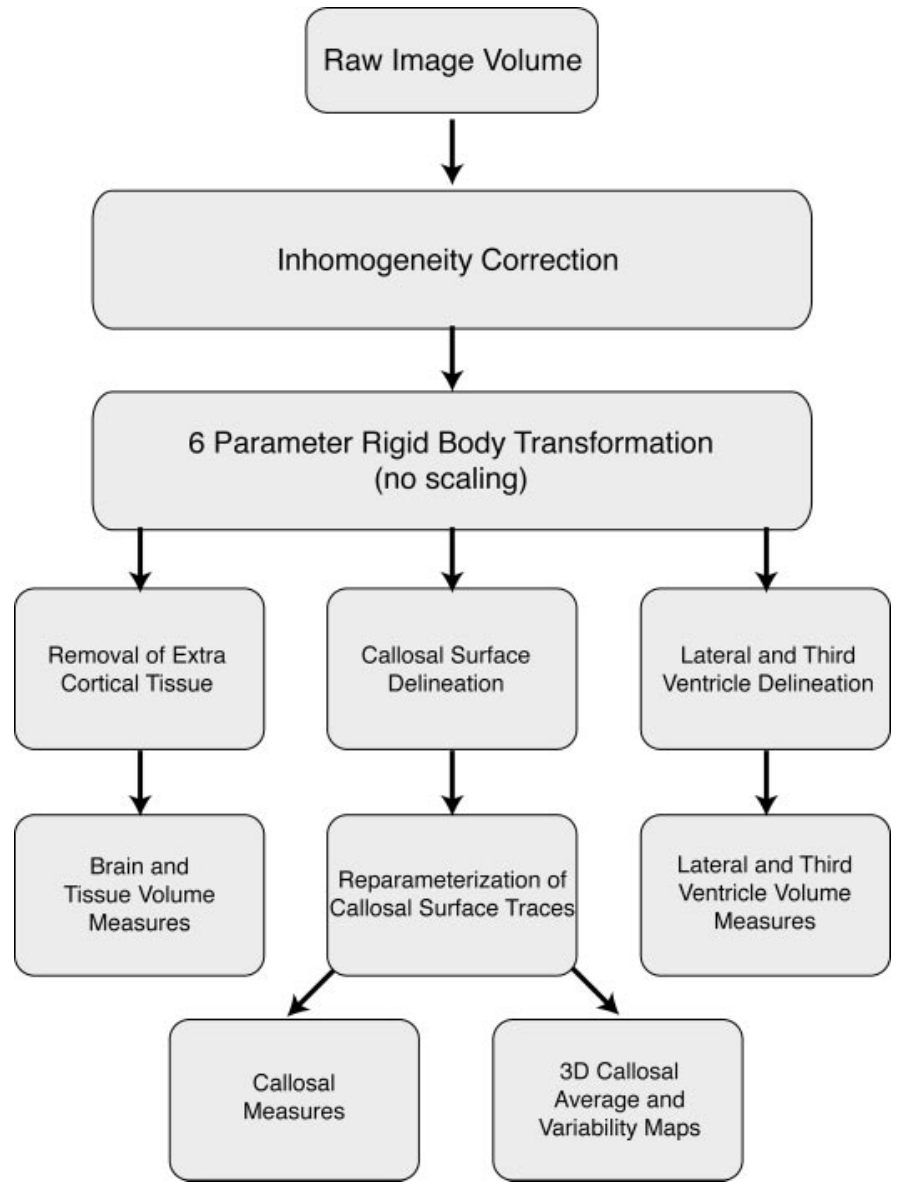

Figure 1. Flow chart summarizing image-processing steps. See Materials and Methods for details.

callosum in each magnified $(4 \times)$ brain volume by following white matter tissue boundaries with a mouse-driven cursor using the software Tracer (Woods, 2001). The segmentation software allowed voxel locations to be recorded at a quarter of a voxel spatial resolution in all three planes (Fig. 2). In the midsagittal plane, the presence of the falx cerebri and septum pellucidum may blur tissue boundaries of the corpus callosum. Therefore, to obtain the most accurate possible measure of midsagittal callosal area, the three most medial brain slices were included $(1 \mathrm{~mm}$ slice thickness). The surface-based mesh modeling approach described below was used to quantify intrarater reliability of callosal surface delineation. The same rater (K.N.) repeatedly outlined the corpus callosum from one randomly chosen brain. Each callosal surface contour, made up of many digitized points, was reparameterized to make these digitized points spatially uniform. The discrepancies in the coordinate locations from spatially equivalent points from each callosal surface tracing were then measured by calculating the root mean square distance (RMS) between corresponding points from each callosal surface. Contouring RMS error was between 0 and $1 \mathrm{~mm}$ at any point on the callosal surface boundary. To ensure the validity of the callosal area measurements, inter-rater reliability was established between two different investigators (K.N. and S.K.). Here the corpus callosum was outlined in six different randomly selected brain volumes. Intraclass correlation coefficients for midsagittal area measures were $r_{\mathrm{I}}=0.96$.

Surface mesh averaging. Surface meshes were constructed from corpus callosum tracings using a surface-based anatomical mesh modeling approach as previously detailed (Thompson et al., 1996a,b, 1997; Narr et al., 2000a). Briefly, as mentioned above, the digitized points representing the callosal surface traces, obtained by manually moving the mouse-driven cursor along the callosal surface boundaries on a computer screen, were made spatially uniform. That is, digitized points making up the callosal surface traces remained in the same coordinate space as each brain volume, but were resampled to make them equal in number. The spatial frequency of points within and across slices was equalized to form a

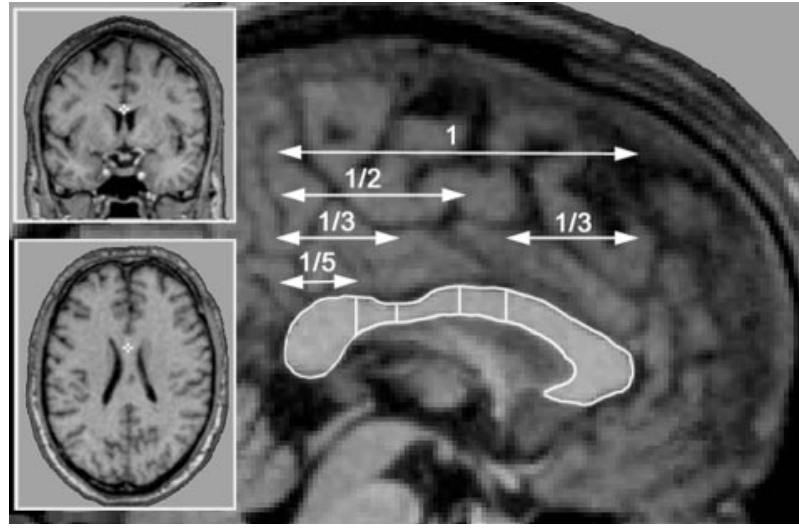

Figure 2. Delineation and partitioning of the midsagittal corpus callosum in T1-weighted MR images. The corpus callosum was traced in the three most medial brain slices. The partitioning scheme adapted from Witelson (1989) and Clarke and Zaidel (1994) was used to divide callosal areas into the following: (1) anterior third; (2) anterior midbody; and (3) posterior midbody, both representing one sixth of callosal area; (4) the isthmus, representing two-fifteenths; and the splenium, representing the posterior fifth of callosal area as shown.

regular parametric grid (Narr et al., 2000a). Homologous grid points from callosal surfaces belonging to members from each group were then matched to obtain average parametric meshes of the corpus callosum. The amount of variation between homologous points from each callosal surface, as compared with the group averages, was calculated on a point-by-point basis to provide spatially detailed intragroup maps of callosal surface variability. This measure of variability was indexed in color and mapped onto the intragroup callosal surface average.

Corpus callosum measures, reported in millimeters, included length, curvature, and two-dimensional surface extremes. These were obtained from the uniformly redigitized grid points representing the callosal surfaces in pixel coordinates in the coordinate space of the ICBM-305 average brain (Mazziotta et al., 1995). These measures thus reflect the native parameters of the callosum in each individual, given that brains were reoriented, but not scaled. That is, the resampling of surface points does not change coordinate locations except to render them equally spaced. Midsagittal areas, reported in square millimeters, were obtained by averaging callosal areas from the three medial brain slices. Callosal areas were further divided into five vertical partitions, based on callosal length, modified from the protocol developed by Witelson (1989) as previously described (Clarke and Zaidel, 1994; Narr et al., 2000) (Fig. 2). These partitions represent callosal channels from (1) the anterior third of the corpus callosum, (2) the anterior body, (3) the posterior body, (4) the isthmus, and (5) the splenium.

Brain and ventricle volume. Brain tissue volumes and lateral and third ventricle volumes were obtained after classifying each image set into tissue types that included gray matter, white matter, CSF, and background after removal of extracortical tissue, as previously described (Sowell et al., 1999; Narr et al., 2000a; Thompson et al., 2001b). Ventricular volumes, including choroid plexus, were obtained by outlining ventricular tissue boundaries in consecutive coronal slices while viewing orthogonal planes also detailed previously (Narr et al., 2000a, 2001). To establish inter-rater reliability, two investigators (K.N. and S.K.) delineated the ventricles in six randomly chosen brain volumes. Inter-rater reliability between raters for total ventricular volume measures was $r_{\mathrm{I}}=$ 0.96 .

Brain size correction. We chose to examine corpus callosum parameters both with and without brain size correction in our statistical analyses, given that relationships between callosal size and brain size may differ across biological risk groups and our goal was to target differences specific to the corpus callosum. Furthermore, correlations between brain volume and callosal parameters could be genetically mediated and thus differ in genetically related versus unrelated individuals. For example, brain size correction in DZ twin pairs or unrelated individuals might be expected to reduce noise by partially correcting for genetically mediated global influences on brain size, whereas brain size correction in MZ twin pairs (where no such genetic differences exist) might only add noise. Because we were interested in comparisons both within genetically 
identical groups and across genetically disparate groups, we report statistical results using both raw callosal measures and callosal measures residualized for brain volume. To clarify the relationships between callosal parameters between co-twins, we have computed intraclass correlation coefficients and corresponding confidence intervals for total midsagittal callosal areas from the four subgroups defined by biological risk.

Procedures for brain size correction differ from our earlier study of callosal morphology, where the scaling of anterior and posterior commissure distances were simultaneously used align callosal morphology for averaging procedures and for brain size corrections in statistical analyses, given that scaling measures, rather than raw brain volumes, showed significant correlations with the dependent variables (Narr et al., 2000a). In the present study, we chose to assess corpus callosum parameters after brain volumes were aligned into a standard orientation with no scaling and therefore to use a brain size correction for the derived parameters in statistical analyses for the reasons described above. It is important to note, however, that our previous methods are expected to reduce variance around anterior and posterior commissure points. The current method may reduce variance obtained from the most rostral and caudal points of the midsagittal callosum, given that these points were included as landmarks for aligning each brain volume. Notwithstanding, both methods serve to register midsagittal anatomy with minimal error, as demonstrated by surface maps of the corpus callosum in 3-D space.

Statistical analyses. Statistical analyses were performed to investigate schizophrenia effects and schizophrenia-associated genetic and nongenetic influences on corpus callosum morphology (K. L. Narr, T. G. M. van Erp, T. D. Cannon, R. P. Woods, P. M. Thompson, S. Jang, R. Blanton, V.-P. Poutanen, M. Huttunen, J. Lönnqvist, C.-G. Standerksjöld-Nordenstam, J. Kaprio, J. C. Mazziotta, and A. W. Toga, unpublished observations). In addition, given that callosal displacements in schizophrenia have been shown to interact with sex (Narr et al., 2000a), schizophrenia effects were assessed separately in male and female diagnostic groups. Corpus callosum morphometric parameters used as dependent measures included (1) total midsagittal areas; (2) areas of the five discrete callosal subpartitions; (3) lengths; (4) heights; (5) dorsal and ventral extremes of the inferior and superior callosal surfaces; and (6) surface curvature.

We chose to analyze this data using $t$ tests given that correlations between measures obtained from monozygotic and dizygotic co-twins may violate the assumptions made by traditional ANOVA models. Moreover, given the size of our sample, we were concerned to minimize the estimation of unnecessary parameters and to maximize the use of our data. Dependent measures were used in paired $t$ tests to examine differences (1) between MZ affected and unaffected discordant co-twins; and (2) between DZ affected and unaffected discordant co-twins. Dependent measures were used in unequal variance $t$ tests to examine differences (3) between schizophrenia patients and controls; (4) between male and female diagnostic groups; (5) between MZ unaffected co-twins and control twins; and (6) between DZ unaffected co-twins and control twins. Unequal variance $t$ tests were also used to examine schizophrenia effects for intracranial and lateral and third ventricle volumes. Relationships between vertical displacement of the corpus callosum and lateral and third ventricular enlargements were assessed using Pearson correlation coefficients.

In all analyses involving control twins (i.e., comparisons 3-6), values from control twin pairs were averaged across both twins for comparisons with schizophrenia probands or their unaffected co-twins. Averaging was performed to avoid the arbitrary selection of one twin for analysis. The use of values averaged across normal twin pairs should reduce variance as compared with measures made on individuals. Furthermore, the use of averages for the controls circumvents the need to explicitly model the different covariances present between $\mathrm{MZ}$ and DZ pairs, given that genetically influenced measures from MZ twins are more likely to be correlated than measures from DZ twins, which are in turn more likely to be correlated than measures from unrelated individuals. Averaging does provide a theoretical expectation of unequal variances, hence the corresponding use of unequal variance $t$ tests in these comparisons. Furthermore, based on our earlier study, we expected that variability in surface parameters may be larger in schizophrenia groups (Narr et al., 2000a).

Measures reflecting callosal surface displacements, including dorsal and ventral callosal surface extremes, surface curvature, and height of the corpus callosum were considered one-tailed hypotheses based on our previous findings. These support a unidirectional displacement of the callosum in schizophrenia (Narr et al., 2000a). Results are reported using one-tailed $p$ values without correction for multiple comparisons. If the measures were uncorrelated, they could be Bonferroni corrected by multiplying these reported $p$ values by five to account for the number of comparisons tested (including callosal length measures). This criterion for significance however, is too stringent, because measures reflecting callosal surface displacements are conceptually as well as statistically correlated (average, $r=0.74$; range, $0.96-0.63$ ). Only measures showing significant schizophrenia effects were examined in follow-up tests of sex, genetic, and environmental influences. $p$ values for callosal area measures reflect two-tailed tests with $p<0.05$ as the threshold for significance. Area measures were not expected to show schizophrenia effects based on our previous findings in an independent sample (Narr et al., 2000a). Additional exploratory tests on subdivisions of callosal area were performed to address isolated reports in the literature of regional area differences even in the absence of global area differences between diagnostic groups. $p$ values are reported for tests both with and without brain size correction. This was done to address the potential differences of brain size correction in comparisons between related versus unrelated individuals as described above, as well as to address the plurality of methods applied by other groups.

To further dissociate the potential influences of genetic and nongenetic factors, the last comparison exclusively compared callosal parameters indexing vertical displacements (superior extremes of the dorsal and ventral callosal surfaces) in MZ and DZ discordant twin pairs, given that callosal displacements in schizophrenia was our primary hypothesis. Here, a nonparametric test was used, counting the number of twin pairs where corpus callosum displacements were larger in the schizophrenia twin, and the number of pairs where displacements were smaller in the schizophrenia twin (Narr, van Erp, Cannon, Woods, Thompson, Jang, Blanton, Poutanen, Huttunen, Lönnqvist, Standerksjöld-Nordenstam, Kaprio, Mazziotta, and Toga, unpublished observations). A two by two contingency table was then prepared, subdividing these counts by zygosity and analyzed using a two-tailed Fisher's exact test.

\section{RESULTS}

\section{Intraclass correlation coefficients and brain size-callosal size correlations}

Brain size and midsagittal callosal area were highly correlated (Pearson $r=0.42 ; p<0.0001$ ). The relationships between cotwins for brain volume, midsagittal callosal area, and brain sizecorrected midsagittal area are shown in Table 2. Intraclass correlation coefficients indicate the magnitude of similarities between MZ and DZ control and discordant co-twins. Increased genetic control is assumed when measures between MZ co-twins are more highly correlated than those between DZ co-twins. As expected, after brain size correction, intraclass correlations for midsagittal areas improve between DZ co-twins, but remain

Table 2. Intraclass correlation coefficients between twin pairs

Discordant twin pairs $r_{1}(95 \%$ confidence intervals)

\begin{tabular}{lll}
\cline { 2 - 3 } Subjects & $\mathrm{MZ}(n=20)$ & $\mathrm{DZ}(n=20)$ \\
\hline Brain volume & $0.81(0.44-0.95)$ & $0.64(0.09-0.90)$ \\
Midsagittal callosal area & $0.90(0.76-0.96)$ & $0.09(0.00-0.05)$ \\
Brain adjusted callosal area & $0.91(0.78-0.96)$ & $0.33(0.00-0.67)$
\end{tabular}

Brain adjusted callosal area
Control twin pairs $r_{1}(95 \%$ confidence intervals $)$

\begin{tabular}{ll}
\hline $\mathrm{MZ}(n=20)$ & $\mathrm{DZ}(n=20)$ \\
$0.90(0.66-0.97)$ & $0.62(0.05-0.89)$ \\
$0.72(0.41-0.88)$ & $0.70(0.37-0.87)$ \\
$0.54(0.13-0.79)$ & $0.74(0.44-0.89)$
\end{tabular}




\begin{tabular}{|c|c|c|c|c|c|}
\hline Effect & Mean $\pm \mathrm{SD}$ & & df & $t$ & $p$ \\
\hline \multicolumn{6}{|c|}{ Schizophrenia patients $(n=20)$ versus normal controls $(n=40)$} \\
\hline Height & SZ: $29.04 \pm 3.18$ & $\mathrm{NC}: 27.21 \pm 2.55$ & 36.3 & 2.00 & $<0.02$ \\
\hline Ventral surface superior boundary & SZ: $91.44 \pm 1.90$ & $\mathrm{NC}: 88.87 \pm 2.23$ & 37.1 & 3.92 & $<0.0002$ \\
\hline Brain size corrected & SZ: $91.19 \pm 1.94$ & $\mathrm{NC}: 88.90 \pm 2.07$ & 37.8 & 3.44 & $<0.0005$ \\
\hline Ventral surface curvature & SZ: $1.59 \pm 0.10$ & $\mathrm{NC}: \quad 1.52 \pm 0.07$ & 34.5 & 2.13 & $<0.02$ \\
\hline Dorsal surface superior boundary & SZ: $96.69 \pm 2.33$ & $\mathrm{NC}: 94.48 \pm 2.24$ & 38 & 3.05 & $<0.002$ \\
\hline Brain size corrected & SZ: $96.42 \pm 2.33$ & NC: $94.61 \pm 2.00$ & 37.2 & 2.63 & $<0.005$ \\
\hline \multicolumn{6}{|c|}{ Male patients $(n=10)$ versus male controls $(n=20)$} \\
\hline Height & SZ: $29.70 \pm 2.41$ & $\mathrm{NC}: 26.93 \pm 2.29$ & 18 & 2.62 & $<0.005$ \\
\hline Brain size corrected & SZ: $28.02 \pm 2.55$ & $\mathrm{NC}: 26.74 \pm 2.21$ & 17.6 & 2.13 & $<0.02$ \\
\hline Ventral surface superior boundary & SZ: $92.62 \pm 1.53$ & $\mathrm{NC}: 89.87 \pm 2.27$ & 15.8 & 3.86 & $<0.0005$ \\
\hline Brain size corrected & SZ: $91.43 \pm 2.19$ & NC: $88.94 \pm 1.91$ & 17.6 & 2.71 & $<0.005$ \\
\hline Ventral surface curvature & SZ: $1.60 \pm 0.08$ & $\mathrm{NC}: \quad 1.53 \pm 0.07$ & 17.6 & 2.23 & $<0.02$ \\
\hline Dorsal surface superior boundary & SZ: $98.12 \pm 1.71$ & $\mathrm{NC}: 94.77 \pm 2.42$ & 16.2 & 3.57 & $<0.001$ \\
\hline Brain size corrected & SZ: $96.86 \pm 2.33$ & NC: $94.41 \pm 1.98$ & 17.6 & 2.53 & $<0.01$ \\
\hline \multicolumn{6}{|c|}{ Female patients $(n=10)$ versus female controls $(n=20)$} \\
\hline Ventral surface superior boundary & SZ: $90.25 \pm 1.45$ & NC: $88.47 \pm 2.22$ & 15.5 & 2.11 & $<0.02$ \\
\hline Brain size corrected & SZ: $90.93 \pm 1.73$ & NC: $89.06 \pm 2.33$ & 16.6 & 2.10 & $<0.02$ \\
\hline \multicolumn{6}{|c|}{ Dizygotic affected $(n=10)$ versus dizygotic unaffected co-twins $(n=10)$} \\
\hline Ventral surface superior boundary & SZ: $91.63 \pm 1.83$ & $\mathrm{NC}: 88.92 \pm 2.60$ & 9 & 5.39 & $<0.0002$ \\
\hline Brain size corrected & SZ: $91.59 \pm 1.70$ & $\mathrm{NC}: 89.45 \pm 2.33$ & 9 & 3.07 & $<0.005$ \\
\hline Dorsal surface superior boundary & SZ: $97.10 \pm 1.99$ & $\mathrm{NC}: 94.47 \pm 2.49$ & 9 & 6.90 & $<0.0001$ \\
\hline Brain size corrected & SZ: $97.06 \pm 2.04$ & $\mathrm{NC}: 95.03 \pm 2.32$ & 9 & 3.04 & $<0.005$ \\
\hline \multicolumn{6}{|c|}{ Monozygotic unaffected co-twins $(n=10)$ versus normal control twins $(n=20)$} \\
\hline Ventral surface superior boundary & SZ: $92.02 \pm 3.92$ & $\mathrm{NC}: 88.47 \pm 2.60$ & 13.5 & 2.54 & $<0.01$ \\
\hline Brain size corrected & SZ: $91.50 \pm 3.64$ & $\mathrm{NC}: 88.64 \pm 1.99$ & 14 & 2.17 & $<0.02$ \\
\hline Dorsal surface superior boundary & SZ: $97.05 \pm 4.00$ & NC: $93.92 \pm 2.39$ & 14.6 & 2.10 & $<0.02$ \\
\hline Brain size corrected & SZ: $96.50 \pm 3.65$ & $\mathrm{NC}: 94.14 \pm 2.18$ & 14.7 & 1.76 & $<0.04$ \\
\hline
\end{tabular}

*All $p$ values in this table reflect one-tailed probability values without correction for multiple comparisons.

primarily unchanged between MZ co-twins. Notwithstanding, large $95 \%$ confidence intervals suggest that intraclass correlations are not a sensitive measure for identifying genetic effects in small sample sizes in which only one or two outliers may strongly influence results.

\section{Brain and ventricle volumes}

Schizophrenia effects were absent for total intracranial, gray, and white matter volumes. Significant CSF volume increases, however, were observed in schizophrenia patients compared with controls $\left(t_{(26.1)}=3.63 ; p<0.001\right.$; mean \pm SEM: patients $=$ $149.6 \pm 47.8 \mathrm{~cm}^{3}$; controls $\left.=107.1 \pm 21.0 \mathrm{~cm}^{3}\right)$. Lateral ventricle enlargements $\left(t_{(35.4)}=3.74 ; p<0.0006\right.$; mean \pm SEM: patients $=$ $16.4 \pm 5.2 \mathrm{~cm}^{3}$; controls $=11.0 \pm 3.9 \mathrm{~cm}^{3}$ ), and third ventricle enlargements $\left(t_{(30.4)}=3.02 ; p<0.005 ;\right.$ mean \pm SEM: patients $=$ $1818.8 \pm 692.7 \mathrm{~mm}^{3}$; controls $=1278.7 \pm 399.4 \mathrm{~mm}^{3}$ ) were present in schizophrenia patients compared with controls.

\section{Corpus callosum morphometric parameters}

Table 3 summarizes significant results from $t$ tests of the callosal parameters described in Materials and Methods (both before and after brain size correction) in groups defined by diagnosis, sex, and/or biological risk for schizophrenia. For measures indexing callosal displacements, one-tailed $p$ values are reported, given our unidirectional hypothesis for this effect in schizophrenia. Group differences were absent for total or partitioned midsagittal callosal areas between schizophrenia patients and their biological rela- tives compared with controls, irrespective of brain size correction. Callosal parameters considered the best predictors of vertical displacement (i.e., superior boundaries of the dorsal and ventral callosal surfaces), showed significant schizophrenia effects that were more pronounced in male diagnostic groups, again both with and without controlling for brain size. Figure 3 illustrates the effects of diagnosis within male and female groups in which native callosal surface averages from each subgroup are superimposed in each sex. Finally, callosal heights and surface curvature were significantly increased in schizophrenia patients versus controls, providing further support for an upward bowing of the corpus callosum in schizophrenia.

Displacements of the superior boundaries of the dorsal and ventral callosal surfaces were also significant in affected versus unaffected DZ co-twins, irrespective of brain size corrections. More interestingly, however, unaffected MZ co-twins of the schizophrenia probands showed the same callosal displacement effects compared with control co-twins, suggesting genetic factors contribute to altered callosal morphology in schizophrenia. These results were less robust after brain size correction, although brain size corrections could potentially add noise in comparisons between MZ co-twins. Callosal parameters did not differ significantly between MZ discordant co-twins, providing no clear evidence of disease-specific differences in callosal morphology. Maps of average native corpus callosum surface representations in patient and biological risk groups illustrate the results shown in 


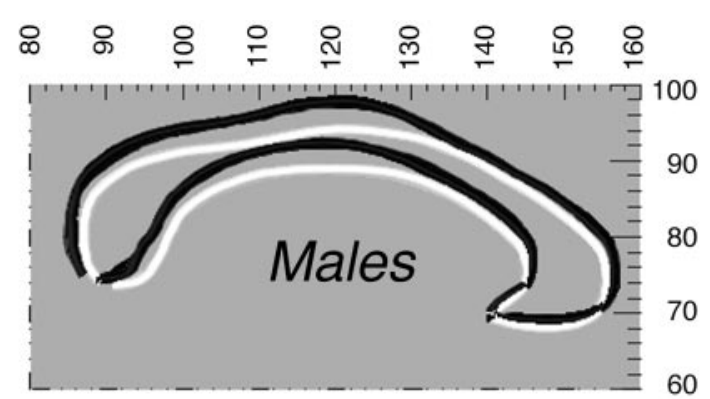

Male schizophrenia patients $(n=10)$ Male normal controls $(n=20)$

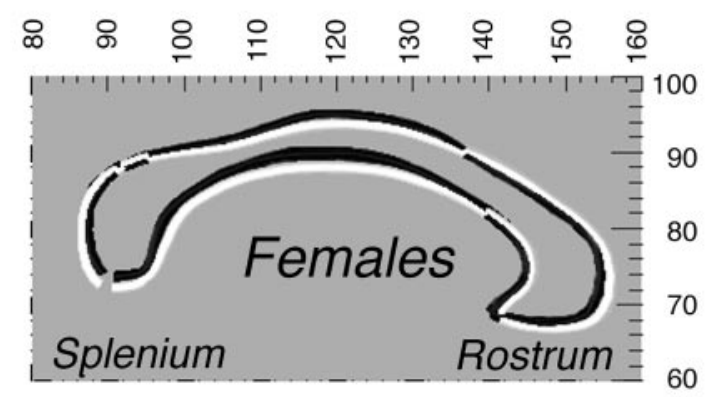

\section{Female schizophrenia patients $(n=10)$ Female normal controls $(n=20)$}

Figure 3. Callosal surface averages mapped in diagnostic groups defined by sex. The average parametric midsagittal mesh models of the corpus callosum are superimposed in male and female groups in the coordinate space of the ICBM 305 average brain where patients are shown in black, and normal controls are shown in white.

Table 3 where genetic, but not disease-specific effects appear to contribute to callosal displacement in schizophrenia (Fig. 4).

To further dissociate genetic versus nongenetic contributions to displacements of corpus callosum morphology in schizophrenia, Figure 5 plots the raw superior dorsal and ventral callosal boundaries for schizophrenia probands against the same measures from their unaffected siblings. For superior dorsal surface boundaries among MZ pairs, five are below the line of identity, indicating increased vertical displacement in the proband, and five are above. In the DZ pairs, all 10 pairs are below the line of identity. This difference in distribution is significant by a twotailed Fisher's exact test $(p<0.03)$, confirming the influence of disease genes on superior surface displacement. This effect was not significant for the superior boundary of the ventral callosal surface or for either surface after brain size correction (all $p>$ $0.14)$.

\section{Corpus callosum variability maps}

Variability maps show the means and variability within each group for corresponding coordinate point locations along the entire callosal surface in 3-D, reflecting quantitative information but not statistical significance. Corpus callosum surface variability mapped separately in discordant and control MZ and DZ co-twins, with control pairs randomly split, appears greatest in the unaffected co-twins of the schizophrenia probands (Fig. 6). Both inferior and superior surface variability are greater in MZ unaffected co-twins of the schizophrenia proband, whereas supe-

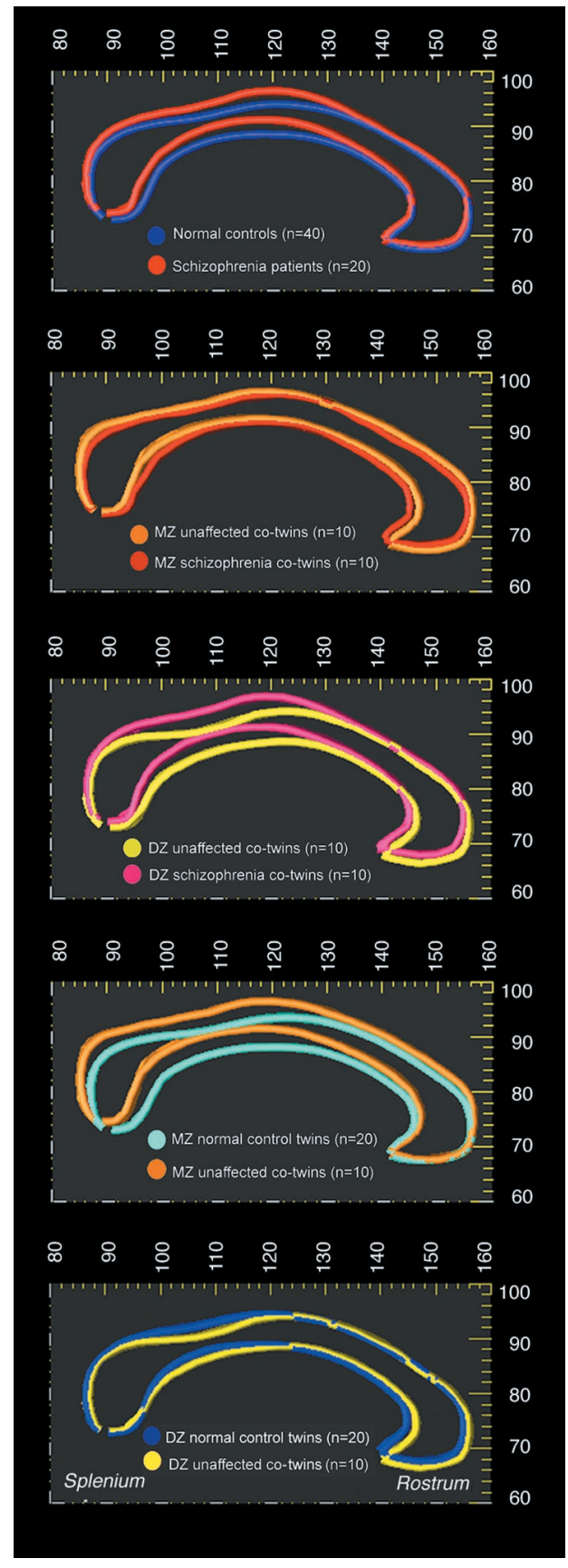

Figure 4. Callosal surface averages mapped in groups defined by biological risk for schizophrenia. Average anatomical mesh models of the corpus callosum are shown in different colors to illustrate differences between groups as measured in the five statistical tests used to establish schizophrenia, genetic, and nongenetic influences on callosal morphology. From the top midsagittal callosal averages are mapped in the following: (1) schizophrenia patients and controls; (2) unaffected and affected monozygotic $(M Z)$ co-twins; (3) unaffected and affected dizygotic $(D Z)$ co-twins; (4) unaffected $\mathrm{MZ}$ co-twins of the schizophrenia probands and MZ control twin pairs; and (5) unaffected DZ co-twins of the schizophrenia probands and DZ control twin pairs.

rior surface variability is increased in the unaffected DZ co-twins. Interestingly, variability in callosal midbody regions in $\mathrm{MZ}$ and DZ schizophrenia subgroups, where callosal displacement may be greatest, exhibit only slightly more surface variability compared with control co-twins. 

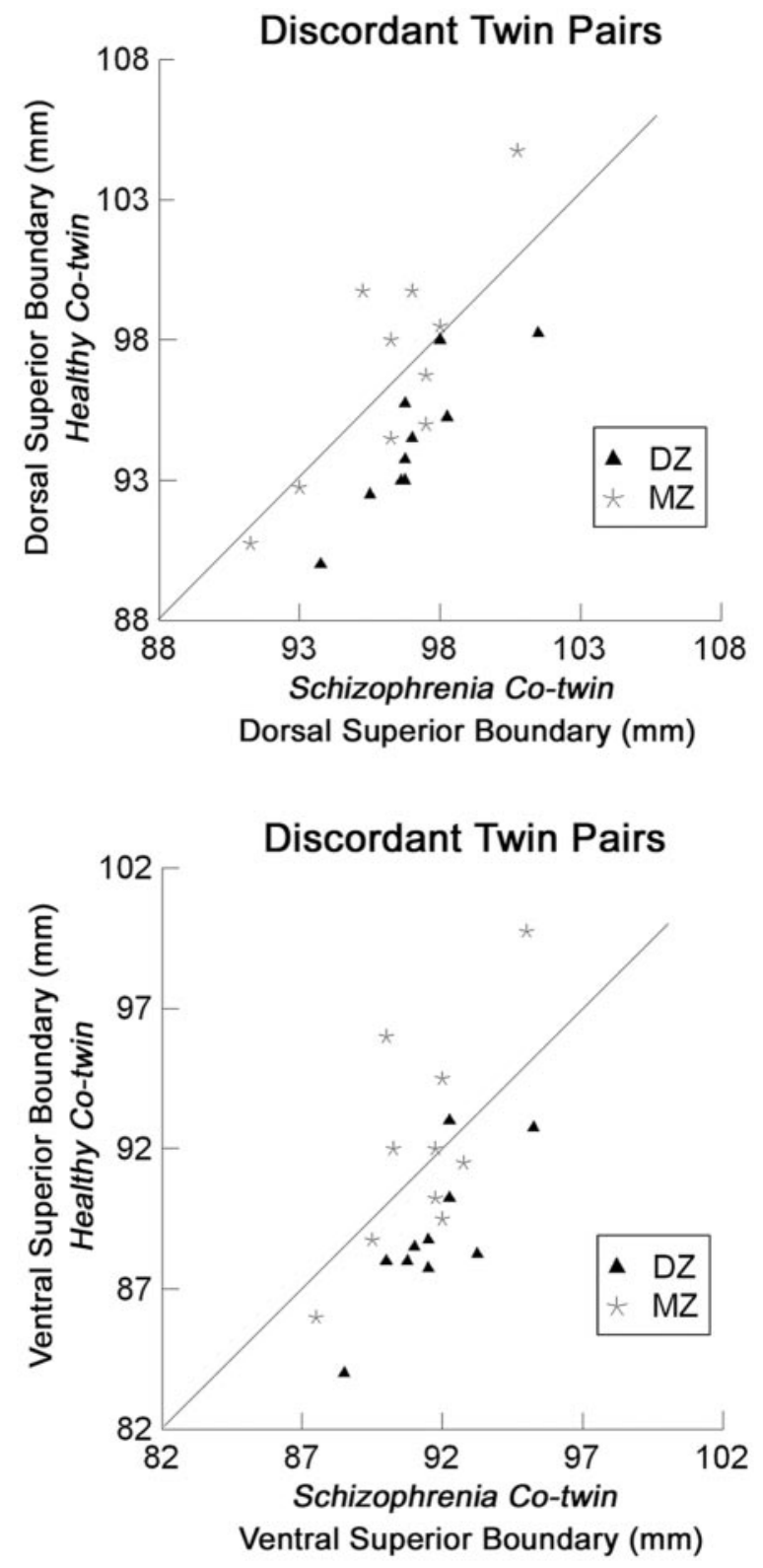

Figure 5. Similarities between discordant MZ and DZ co-twins for superior dorsal surfaces boundaries of the corpus callosum (top) and superior boundaries of the ventral callosal surfaces (bottom). Affected MZ and DZ discordant co-twins are plotted on the $x$-axis, and their healthy co-twins are plotted on the $y$-axis.

\section{Correlations between callosal displacement and ventricular volumes}

Correlation analyses were performed between the superior boundaries of the dorsal and ventral callosal surfaces and third and lateral ventricular volume (Fig. 7). Superior boundaries of the dorsal and ventral callosal surfaces were significantly correlated with lateral ventricle volume across all biological risk subgroups (Pearson $r=0.67, r=0.55$, respectively; $p<0.00001$ ). Dorsal and ventral callosal surface superior boundaries were also significantly correlated with third ventricle volume $(r=0.35, r=0.26$; $p<0.001, p<0.02$, respectively) across all risk groups. Within risk groups, these relationships were only significant in normal controls for lateral ventricle volume $(r=0.57, r=0.43 ; p<$ $0.0001, p<0.005$ ), and in unaffected MZ co-twins of the schizo-
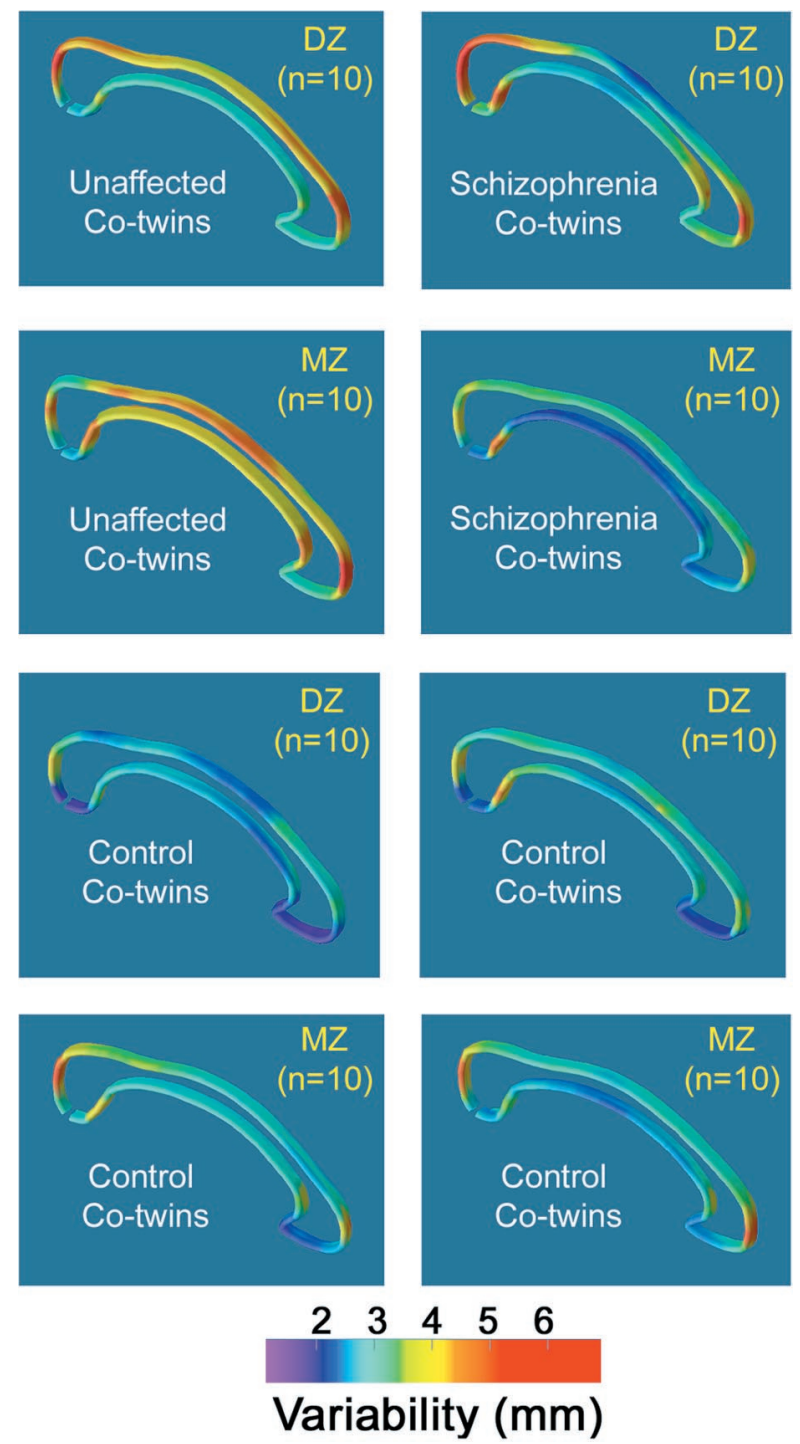

Figure 6. Callosal surface variability mapped separately in discordant and control monozygotic $(M Z)$ and dizygotic $(D Z)$ co-twins. The color bar encodes the root mean square magnitude (in millimeters) of the displacement vectors required to map equivalent surface points from each individual callosal surface to the group average.

phrenia proband $(r=0.74, r=0.62 ; p<0.01, p<0.05)$ for dorsal and ventral superior boundaries, respectively. Relationships were similar for dependent measures after brain size correction.

\section{DISCUSSION}

Vertical displacements of the corpus callosum were present in patients, irrespective of zygosity, compared with controls, confirming our earlier findings (Narr et al., 2000a) and those of other studies supporting an upward bowing of the corpus callosum in schizophrenia (Casanova et al., 1990a,b; DeQuardo et al., 1996; Frumin et al., 1998). Furthermore, by examining differences between MZ and DZ discordant and control co-twins, we demonstrated that genetic factors contribute to callosal displacement in schizophrenia. These results suggest that callosal shape differences are an easily identifiable neuroanatomic marker by which to separate individuals possessing a genetic vulnerability for developing schizophrenia. It is important to note, however, that in the embryology of MZ twinning there are two major types of pla- 

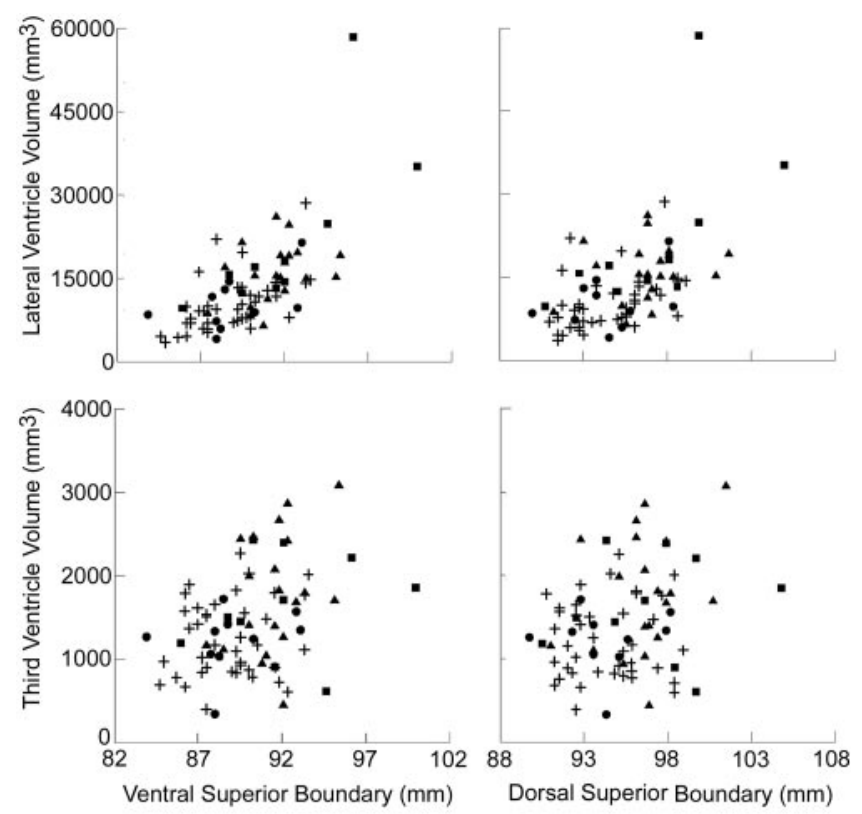

- Schizophrenia co-twins ( $(n=20) \quad-$ Unaffected DZ co-twins $(n=10)$ - Unaffected MZ co-twins ( $n=10)+$ Normal control co-twins $(n=40)$

Figure 7. Correlations between the superior boundaries of the dorsal and ventral callosal surfaces and third and lateral ventricular volume in groups defined by biological risk for schizophrenia. Dorsal (right) and ventral (left) superior boundaries of the corpus callosum are plotted on the $x$-axes in millimeters, and lateral ventricular volumes (top) and third ventricular volumes (bottom) are plotted on the $y$-axes in cubic millimeters.

centation where the sharing of a chorion may make MZ twins more similar or more different compared with twins not sharing a chorion (Prescott et al., 1999). MZ and DZ schizophrenia co-twins, however, did not differ significantly in brain volumes or in callosal displacement measures (data not shown), suggesting that differences in placentation did not influence the results or conclusions made here.

\section{Callosal size in schizophrenia}

We found little evidence for decreased or increased midsagittal callosal areas in schizophrenia patients relative to controls. These findings add little clarity to discrepancies in earlier results where increases (Nasrallah, 1986; Uematsu and Kaiya, 1988) and decreases (Rossi et al., 1989; Stratta et al., 1989; Woodruff et al., 1993; Hoff et al., 1994; Tibbo et al., 1998) in callosal size are reported. Limited control of factors such as sex, handedness, and brain size, previously shown to influence callosal morphology (Witelson, 1985, 1989; Rauch and Jinkins, 1994; Jäncke et al., 1997; Davatzikos and Resnick, 1998; Bermudez and Zatorre, 2001) may be responsible for differences in results. If, as shown in the meta-analyses by Woodruff et al. (1995), effect sizes for reductions in midsagittal area between diagnostic groups are extremely small $(d=0.18)$, with power at 0.80 , sample sizes would have to be $>n=400$ to detect area differences between diagnostic groups. Estimated effect sizes for diagnostic group differences in midsagittal areas in this study were only slightly larger for raw callosal midsagittal areas $(d=0.26)$ and for brain size-corrected areas $(d=0.43)$.

\section{Sex}

Sex differences in callosal morphology remain controversial in normal populations (DeLacoste-Utamsing and Holloway, 1982;
Holloway et al., 1993; Bishop and Wahlsten, 1997; Jäncke et al., 1997; Davatzikos and Resnick, 1998; Bermudez and Zatorre, 2001). Sex differences in callosal size appear similarly complicated in schizophrenia (Narr et al., 2000a). Moreover, callosal fiber densities in all callosal regions, except the posterior midbody and splenium, are reported as greater in female compared with male subjects, with these relationships across sex reversed in schizophrenia (Highley et al., 1999). In our study, diagnostic differences in callosal area were not present in male or female groups. Recent studies using larger study groups do not clarify sex by diagnostic group interactions for callosal size. For example, one study found significantly reduced callosal size in male patients compared with controls (females were not studied) (Tibbo et al., 1998). In contrast, a second study reported larger anterior callosal regions in typical onset females compared with controls, with no differences exhibited between male groups (SchellerGilkey and Lewine, 1999). Disparities in findings may persist because of differences in the brain size correction procedures. For example, Bermudez and Zatorre (2001) have shown that sex effects in callosal morphology vary according to the brain normalization strategies used, rendering many studies incomparable.

Callosal displacements were, as previously reported (Narr et al., 2000a), more pronounced between male diagnostic groups, although female patients exhibited significant vertical displacement of ventral callosal surfaces. Sex differences across diagnostic groups may reflect sexual dimorphisms in schizophrenia phenomenology, in which male patients show increased negative symptoms, earlier age of onset, and a worse course of illness compared with females (DeLisi et al., 1989; Gur et al., 1996). Increased displacements of the callosum, however, may link more directly with symptom severity, as previously shown for ventricular enlargements (Shenton et al., 2001). Impractically small sample sizes prohibited the examination of sex differences separately in biological risk groups in this study.

\section{Disease-specific effects}

Two related studies have addressed whether differences in callosal size exist between MZ twins discordant for schizophrenia (Casanova et al., 1990a,b). Results were in concordance with our findings, suggesting that disease-specific environmental influences are absent. In contrast with our findings, however, these studies report altered callosal shape in affected MZ co-twins compared with their unaffected co-twins. Notwithstanding, our variability maps (Fig. 6) indicate increased surface variability in discordant MZ twins that perhaps mask very small differences in callosal displacements between co-twins. Maps of callosal surface averages, however, clearly show that unaffected and affected MZ co-twins posses strikingly similar profiles in the midsagittal plane (Fig. 4). Differences in displacements between the discordant DZ co-twins mirrored our reported schizophrenia effects. Because DZ co-twins share on average approximately half of their genes, these results do not distinguish genetic from environmental influences.

\section{Genetic effects}

This is the first study to directly assess genetic and/or shared environmental contributions to callosal size and displacement differences in schizophrenia. Callosal size was not different between unaffected biological relatives of schizophrenia probands compared with controls. Significant displacements of the callosa, however, were observed in unaffected MZ co-twins relative to control twins, suggesting genetic influences. To confirm the pres- 
ence of genetic rather than shared environmental contributors toward callosal surface displacements, we counted discordant co-twins of each zygosity that exhibited greater indices of callosal displacement. Dorsal surface displacements were always present in the affected DZ co-twins relative to their healthy siblings, whereas this was true only half the time in MZ affected co-twins compared with their healthy siblings (Fig. 5). Genetic rather than shared environmental influences, therefore, appear to be the primary contributors to displacement effects. That is, harmful events occurring in utero, or postnatally to both twins, would cause both discordant co-twins, whether MZ or DZ, to exhibit increased vertical displacements of the corpus callosum compared with controls. Our findings support a prominent role of genetic factors toward changes in anatomy that manifest as a vertical shift of the corpus callosum within the midsagittal section in schizophrenia.

\section{Ventricular enlargements and callosal displacements}

Given that callosal size appears unchanged between patients and controls, an upward bowing of the corpus callosum schizophrenia may be interpreted to reflect differences in ventricle size rather an abnormality intrinsic to function of the corpus callosum. Three studies have shown that ventricular enlargements influence shape and displacements of the callosum in schizophrenia (Casanova et al., 1990a; Downhill et al., 2000; Narr et al., 2000a). Previously in chronic schizophrenia, we found significant relationships between vertical callosal surface displacements and lateral, but not third ventricular enlargement. In a more refined study of ventricular shape, we further demonstrated that vertical displacements of superior and posterior horn ventricular surfaces were highly correlated with callosal displacements and curvature (Narr et al., 2000b), and with significant, but less pronounced cingulate sulcus displacements. Here, we report that both lateral and third ventricle enlargement contribute to callosal displacements in schizophrenia, with lateral ventricular enlargement more highly correlated.

Links between ventricular enlargements and callosal displacements were significant overall, but not in all biological risk groups, attributable potentially to reductions in power resulting from smaller subgroups. Our previous failure to find relationships between callosal displacements and third ventricle enlargements may reflect smaller effects between neuroanatomic regions that are more distal. That is, because the callosum forms the roof of the lateral ventricle superior horn relationships are assumed to be more pronounced. Alternatively, associations between third ventricle enlargements and callosal displacements may reflect primary relationships with disease effects rather than reflective of dorsal shifts in anatomy. As a case in point, vertical displacements of the callosum are highly correlated with decreases in hippocampal volume in the same study group (Narr, van Erp, Cannon, Woods, Thompson, Jang, Blanton, Poutanen, Huttunen, Lönnqvist, Standerksjöld-Nordenstam, Kaprio, Mazziotta, and Toga, unpublished data) where it is unlikely that decreases in hippocampal volume in schizophrenia cause upward shifts in anatomy (Narr, van Erp, Cannon, Woods, Thompson, Jang, Blanton, Poutanen, Huttunen, Lönnqvist, Standerksjöld-Nordenstam, Kaprio, Mazziotta, and Toga, unpublished observations).

It was our goal to examine callosal morphology within the context of the entire brain volume rather than as a separate entity, so that differences relating to abnormalities in surrounding brain morphology would not be obscured. Displacements representing an upward bowing of the corpus callosum were confirmed in schizophrenia and found to be more pronounced between male diagnostic groups. Genetic rather than shared environmental or disease-specific influences contribute to displacements of the corpus callosum, where effects are related to both lateral and third ventricle enlargements. It is suggested, however, that lateral rather than third ventricle enlargements are responsible for an upward shift of the corpus callosum in schizophrenia, although this remains to be clarified in a more refined study of ventricular shape in MZ and DZ co-twins discordant for schizophrenia. Results from this study indicate schizophrenia-related genetic associations with callosal displacement. They may prove useful by providing clinicians and geneticists with an anatomical marker for identifying individuals with a genetic vulnerability for schizophrenia, although they show no symptoms.

\section{REFERENCES}

Bermudez P, Zatorre RJ (2001) Sexual dimorphism in the corpus callosum: methodological considerations in MRI morphometry. NeuroImage 13:1121-1130.

Bishop KM, Wahlsten D (1997) Sex differences in the human corpus callosum: myth or reality? Neurosci Biobehav Rev 21:581-601.

Cannon TD, Kaprio J, Lonnqvist J, Huttunen M, Koskenvuo M (1998) The genetic epidemiology of schizophrenia in a Finnish twin cohort: a population-based modeling study. Arch Gen Psychiatry 55:57-64.

Cannon TD, Thompson PM, van Erp TG, Toga AW, Poutanen V-P, Huttunen M, Lonnqvist J, Standerskjold-Nordenstam C-G, Narr KL, Khaledy M, Zoumalan CI, Dail R, Kaprio J (2002) Cortex mapping reveal heteromodal gray matter deficits in twins discordant for schizophrenia. Proc Natl Acad Sci USA 99:3228-3233.

Casanova MF, Zito M, Goldberg TE, Suddath RL (1990a) Corpus callosum curvature in schizophrenic twins. Biol Psychiatry 28:83-84.

Casanova MF, Sanders RD, Goldberg TE, Bigelow LB, Christison G, Torrey EF, Weinberger DR (1990b) Morphometry of the corpus callosum in monozygotic twins discordant for schizophrenia: a magnetic resonance imaging study. J Neurol Neurosurg Psychiatry 53:416-421.

Chua SE, Sharma T, Takei N, Murray RM, Woodruff PW (2000) A magnetic resonance imaging study of corpus callosum size in familial schizophrenic subjects, their relatives, and normal controls. Schizophr Res 41:397-403.

Clarke JM, Zaidel E (1994) Anatomical-behavioral relationships: corpus callosum morphometry and hemispheric specialization. Behav Brain Res 64:185-202.

Davatzikos C, Resnick S (1998) Sex differences in anatomic measures of interhemispheric connectivity: correlations with cognition in women but not men. Cereb Cortex 8:635-640.

DeLacoste-Utamsing C, Holloway RL (1982) Sexual dimorphism in the human corpus callosum. Science 216:1431-1432.

DeLisi LE, Dauphinais ID, Hauser P (1989) Gender differences in the brain: are they relevant to the pathogenesis of schizophrenia? Comp Psychiatry 30:197-208.

DeQuardo JR (1999) Landmark analysis of corpus callosum shape in schizophrenia. Biol Psychiatry 46:1712-1714.

DeQuardo JR, Bookstein FL, Green WD, Brunberg JA, Tandon R (1996) Spatial relationships of neuroanatomic landmarks in schizophrenia. Psychiatry Res 67:81-95.

Downhill Jr JE, Buchsbaum MS, Wei T, Spiegel-Cohen J, Hazlett EA, Haznedar MM, Silverman J, Siever LJ (2000) Shape and size of the corpus callosum in schizophrenia and schizotypal personality disorder. Schizophr Res 42:193-208.

Frumin M, Golland P, McCarley RW, Hirayasu Y, Salisbury DF, Kikinis R, Shenton M. (1998) Shape differences in first episode schizophrenia and affective disorder, 37th Annual Meeting of the American College of Neuropsychopharmacology, San Juan, Puerto Rico, December.

Gharaibeh WS, Rohlf FJ, Slice DE, DeLisi LE (2000) A geometric morphometric assessment of change in midline brain structural shape following a first episode of schizophrenia. Biol Psychiatry 48:398-405.

Gur RE, Petty RG, Turetsky BI, Gur RC (1996) Schizophrenia throughout life: sex differences in severity and profile of symptoms. Schizophr Res 21:1-12.

Highley JR, Esiri MM, McDonald B, Cortina-Borja M, Herron BM, Crow TJ (1999) The size and fibre composition of the corpus callosum with respect to gender and schizophrenia: a post-mortem study. Brain 122:99-110.

Hoff AL, Neal C, Kushner M, DeLisi LE (1994) Gender differences in corpus callosum size in first-episode schizophrenics. Biol Psychiatry 35:913-919.

Holloway RL, Anderson PJ, Defendini R, Harper C (1993) Sexual dimorphism of the human corpus callosum from three independent 
samples: relative size of the corpus callosum. Am J Phys Anthropol 92:481-498.

Jäncke L, Staiger JF, Schlaug G, Huang Y, Steinmetz H (1997) The relationship between corpus callosum size and forebrain volume. Cereb Cortex 7:48-56.

MacDonald D (1996) MNI-Register: program for interactive volume display, point tagging and defining transformations (http://www.bic. mni.mcgill.ca/software/register/register.html). Mc Connell Brain Imaging Center, Montreal Neurological Institute, Montreal, Canada.

MacDonald D, Avis D, Evan AC (1994) Multiple surface identification and matching in magnetic resonance imaging. Proc Soc Photo-Opt Instr Eng 2359:160-169.

Mazziotta JC, Toga AW, Evans A, Fox P, Lancaster J (1995) A probabilistic atlas of the human brain: theory and rationale for its development (The International Consortium for Brain Mapping: ICBM). NeuroImage 2:89-101.

Mohr B, Pulvermueller F, Cohen R, Rockstroh B (2000) Interhemispheric cooperation during word processing: evidence for callosal transfer dysfunction in schizophrenic patients. Schizophr Res 46:231-239.

Narr KL, Thompson PM, Sharma T, Moussai J, Cannestra AF, Toga AW (2000a) Mapping morphology of the corpus callosum in schizophrenia. Cereb Cortex 10:40-49.

Narr KL, Thompson PM, Sharma T, Moussai J, Khaledy M, Jang S, Eghbalieh S, Toga AW (2000b) 3D atlas of sulcal trajectories and asymmetries in schizophrenic and normal populations: gender effects, 6 th International Conference on Functional Mapping of the Human Brain, San Antonio, Texas, June.

Nasrallah HA (1986) A controlled magnetic resonance imaging study of corpus callosum thickness in schizophrenia. Biol Psychiatry 21:274-282.

Prescott CA, Johnson RC, McArdle JJ (1999) Chorion type as a possible influence on the results and interpretation of twin study data. Twin Res 2:244-249.

Rauch RA, Jinkins JR (1994) Analysis of cross-sectional area measurements of the corpus callosum adjusted for brain size in male and female subjects from childhood to adulthood. Behav Brain Res 64:65-78.

Rossi A, Stratta P, Gallucci M, Passariello R (1989) Quantification of corpus callosum and ventricles in schizophrenia with nuclear magnetic resonance imaging: a pilot study. Am J Psychiatry 146:99-101.

Scheller-Gilkey G, Lewine RR (1999) Age at onset and sex differences in corpus callosum area in schizophrenia. Schizophr Res 40:229-235.

Shenton ME, Dickey CC, Frumin M, McCarley RW (2001) A review of MRI findings in schizophrenia. Schizophr Res 49:1-52.

Sled JG, Pike GB (1998) Standing-wave and RF penetration artifacts caused by elliptic geometry: an electrodynamic analysis of MRI. IEEE Trans Med Imaging 17:653-662.

Sowell ER, Thompson PM, Holmes CJ, Batth R, Jernigan TL, Toga AW (1999) Localizing age-related changes in brain structure between childhood and adolescence using statistical parametric mapping. NeuroImage 9:587-597.

Stratta P, Rossi A, Gallucci M, Amicarelli I (1989) Hemispheric asymmetries and schizophrenia: a preliminary magnetic resonance imaging study. Biol Psychiatry 25:275-284.

Thompson PM, Schwartz C, Toga AW (1996a) High-resolution random mesh algorithms for creating a probabilistic 3D surface atlas of the human brain. NeuroImage 3:19-34.

Thompson PM, Schwartz C, Lin RT, Khan AA, Toga AW (1996b) Three-dimensional statistical analysis of sulcal variability in the human brain. J Neurosci 16:4261-4274.

Thompson PM, MacDonald D, Mega MS, Holmes CJ, Evans AC, Toga AW (1997) Detection and mapping of abnormal brain structure with a probabilistic atlas of cortical surfaces. J Comp Assist Tomogr 21:567-581

Thompson PM, Narr KL, Blanton RE, Toga AW (2001a) Mapping structural alterations of the corpus callosum during brain development and disease, (Iacoboni M, Zaidel E, eds), in press.

Thompson PM, Cannon TD, Narr KL, van Erp T, Poutanen V, Huttunen M, Lonnqvist J, Standerskjold-Nordenstam C, Kaprio J, Khaledy M, Dail R, Zoumalan C, Toga AW (2001b) Genetic influences on brain structure. Nat Neurosci 12:1253-1258.

Tibbo P, Nopoulos P, Arndt S, Andreasen NC (1998) Corpus callosum shape and size in male patients with schizophrenia. Biol Psychiatry 44:405-412.

Uematsu M, Kaiya H (1988) The morphology of the corpus callosum in schizophrenia: an MRI study. Schizophr Res 1:391-398.

Witelson SF (1985) The brain connection: the corpus callosum is larger in left-handers. Science 229:665-668.

Witelson SF (1989) Hand and sex differences in the isthmus and genu of the human corpus callosum. A postmortem morphological study. Brain 112:799-835

Woodruff PW, Pearlson GD, Geer MJ, Barta PE (1993) A computerized magnetic resonance imaging study of corpus callosum morphology in schizophrenia. Psychol Med 23:45-56.

Woodruff PWR, McManus IC, David AS (1995) Meta-analysis of corpus callosum size in schizophrenia. J Neurol Neurosurg Psychiatry 58:457-462.

Woodruff PWR, Phillips ML, Rushe T, Wright IC (1997) Corpus callosum size and inter-hemispheric function in schizophrenia. Schizophr Res 23:189-196.

Woods RP (2001) Tracer: java based 3D-tracing program (http://bishopw. loni.ucla.edu/Tracer/Tracer.html). UCLA Brain Mapping Center, Department of Neurology, University of California at Los Angeles School of Medicine, Los Angeles, CA.

Zijdenbos AP, Dawant BM (1994) Brain segmentation and white matter lesion detection in MR images. Crit Rev Biomed Eng 22:401-465. 